\title{
ASPECTOS RELACIONADOS À OCORRÊNCIA E MECANISMO DE AÇÃO DE FUMONISINAS
}

\author{
OCCURRENCE AND FUMONISINS PATHOPHISIOLOGY-REVIEWS
}

\author{
Claudia Rodrigues Pozzi ${ }^{1}$ Juliana Rodrigues Pozzi Arcaro ${ }^{2}$ \\ Irineu Arcaro Júnior ${ }^{3}$ Helena Fagundes ${ }^{4}$ Benedito Corrêa ${ }^{5}$
}

\section{- REVISÃO BIBLIOGRÁFICA -}

RESUMO

As fumonisinas são micotoxinas produzidas por fungos do gênero Fusarium, um dos principais fitopatógenos de grãos de milho. Isoladas em 1988, a partir de amostras de milho mofado proveniente de região com alta incidência de câncer do esôfago na África, foram relacionadas à ocorrência de leucoencefalomalácia eqüina e hidrotórax e edema pulmonar suíno, e outras patologias animais. A presente revisão aborda aspectos da ocorrência natural das fumonisinas em grãos de milho, características físico-química das moléculas e efeitos toxicológicos em eqüinos, suínos, aves e bovinos, além do provável mecanismo de ação das micotoxinas.

Palavras-chave: Fumonisinas, F.verticillioides (Sacc.) Nirenb.

\section{SUMMARY}

Fumonisins are micotoxins produced by a fungus of the genera Fusarium, one of the main fitopathogens that occur in maize. Isolated in 1988, from moudly corn samples in a high incidence area of esophageal cancer in Africa, fumonisins are related with several diseases including equine leucoencephalomalacia and porcine pulmonary edema and hydrothorax. The present review approaches the natural occurrence of fumonisins in maize, its physical and quimical molecular characteristics and toxicological effects in horses, porcine, poultry and cattle. This review also discusses the micotoxins pathophisiology.
Key words: Fumonisins, F.verticillioides (Sacc.) Nirenb.

\section{INTRODUÇÃO}

Os fungos filamentosos produzem uma imensa diversidade de metabólitos secundários, como pigmentos, antibióticos, ftotoxinas além de compostos tóxicos, denominados de micotoxinas. Quando produzidos em associação com os alimentos, ração animal e forragens, os metabólitos tóxicos podem ser ingeridos pelo homem e animais, provocando as micotoxicoses (MOSS, 1991)

O mais conhecido episódio de micotoxicose ocorreu em 1960, na Inglaterra, quando a torta de amendoim importada do Brasil foi responsabilizada como causadora da "Doença X dos perus". Desta torta, foi obtido um extrato clorofórmico que, ao ser administrado a marrecos jovens, foi capaz de reproduzir as lesões hepáticas semelhantes à doença original, sendo a toxina denominada de A. flavus toxin ou aflatoxina (ALLCROFT et al., 1961; ALLCROFT \& CARNAGHAM, 1962)

\footnotetext{
${ }^{1}$ Médico Veterinário, Pesquisador Científico IV, Doutor, Centro de Nutrição e Alimentação Animal, Instituto deZootecnia, 13460-000, Nova Odessa, SP. E-mail:pozzi@izsp.br. Autor para correspondência.

${ }^{2}$ Médico Veterinário, Pesquisador Científico III, Mestre, Centro de Nutrição e Alimentação Animal, Instituto de Zootecnia, Nova Odessa, SP.

${ }^{3}$ Zootecnista, Pesquidador Científico IV, Doutor, Centro de Etologia e Ambiência, Instituto de Zootecnia.

${ }^{4}$ Medico Veterinário, bolsista Fapesp, Instituto de Zootecnia.

${ }^{5}$ Biomédico, Livre Docente, Departamento de Microbiologia, Instituto de Ciências Biomédicas, Universidade de São Paulo.
} 
Em decorrência dos grandes esforços da comunidade científica internacional, desde 1960 a micotoxicologia tem sido desenvolvida e novas micotoxinas vêm sido isoladas e caracterizadas, especialmente as fusariotoxinas, micotoxinas produzidas pelo gênero Fusarium, dentre as quais se destacam as fumonisinas, que assim como as aflatoxinas, podem ser consideradas de ocorrência mundial.

As espécies do gênero Fusarium são citadas por MILLS (1989) como as principais invasoras de grãos de milho no campo, causando inclusive várias doenças, como a podridão de sementes e colmo. O pesquisador alerta que estas espécies, que invadem a planta no campo, também podem ser encontradas no armazenamento, caso as condições de temperatura e umidade sejam adequadas. O Fusarium verticillioides (Sacc.) Nirenb. é o fungo mais freqüente em grãos de milho recém colhidos, com níveis de contaminação de até $100 \%$. No Brasil, algumas espécies do gênero Fusarium já foram isoladas do milho e outros substratos procedentes de vários Estados brasileiros, com predominância do $\boldsymbol{F}$. verticillioides (Sacc.) Nirenb. (ASEVEDO et al., 1994; POZZI et al., 1995; ORSI et al., 2000; SILVA et al., 2001).

\section{CARACTERÍSTICAS FÍSICO-QUÍMICAS DAS FUMONISINAS}

As fumonisinas são metabólitos fúngicos secundários produzidos por Fusarium verticillioides (Sacc.) Nirenb. (GELDERBLOM et al., 1988; BEZUIDENHOUT et al., 1988). Porém, outras espécies do gênero Fusarium também são produtoras de fumonisinas: Fusarium proliferatum (ROSS et al., 1990), Fusarium nygamai (THIEL et al., 1992), Fusarium anthophilum, Fusarium dlamini e Fusarium napiforme (NELSON, 1992), Fusarium subglutinans (SCOTT, 1993), Fusarium polyphialidicum (ABBAS \& OCAMB, 1995), Fusarium oxysporum (AH SEO \& WON LEE, 1999).

As fumonisinas são moléculas estruturalmente relacionadas e, até o momento, 16 foram isoladas e caracterizadas: Fumonisina $\mathrm{B}_{1}\left(\mathrm{FB}_{1}\right), \mathrm{FB}_{2}$, $\mathrm{FB}_{3}, \mathrm{FB}_{4}, \mathrm{~A}_{1}, \mathrm{~A}_{2}, \mathrm{~A}_{3}, \mathrm{AK}_{1}, \mathrm{C}_{1}, \mathrm{C}_{3}, \mathrm{C}_{4}, \mathrm{P}_{1}, \mathrm{P}_{2}, \mathrm{P}_{3}, \mathrm{PH}_{1 \mathrm{a}}, \mathrm{PH}_{1 \mathrm{~b}}$ (MUSSER \& PLATTNER, 1997; AH-SEO \& WON LEE, 1999). As análises de ressonância nuclear magnética e espectrometria de massa revelaram que a fumonisina $B_{1}$ é um diester de propano 1, 2, 3 ácido tricarboxílico e 2 - amino - 12, 16 dimetil - 3, $5,10,14,15$ - pentahidroxicosano em que nos $\mathrm{C}_{14} \mathrm{e}$ $\mathrm{C}_{15}$ os grupos hidroxilas são esterificados com o grupo carboxiterminal de propano 1, 2, 3 - ácido tricarboxílico (BEZUIDENHOUT et al., 1988).
As fumonisinas chamadas de $\mathrm{FB}_{1}$ e $\mathrm{FB}_{2}$ foram isoladas de cepa $\boldsymbol{F}$. verticillioides (Sacc.) Nirenb. causadora de leucoencefalomalácia eqüina por GELDERBLOM et al. (1988). As estruturas químicas das moléculas foram elucidadas por BEZUIDENHOUT et al. (1988). As fumonisinas $B_{3}$ e $\mathrm{B}_{4}$, bem como os seus produtos de hidrólise (série "HB"), foram caracterizadas por CAWOOD et $\boldsymbol{a l}$. (1991), as fumonisinas da série "A" por BEZUIDENHOUT $\boldsymbol{e}$ t al. (1988) e da série "C" por BRANHAM \& PLATTNER (1993).

As fumonisinas são mo léculas fortemente polares, solúveis em água e em acetonitrila-água e insolúveis em solventes orgânicos. A hidrólise das fumonisinas, através do aquecimento com ácido hidroclórico $6 \mathrm{M}$ ou hidróxido de potássio 0,05 e 2 M produz ácido tricarboxílico e o aminopoliol correspondente (SCOTT, 1993).

\section{OCORRÊNCIA NATURAL DAS FUMONISINAS}

Das fumonisinas identificadas até aqui, as $\mathrm{FB}_{1}, \mathrm{FB}_{2}$ e $\mathrm{FB}_{3}$ são as mais isoladas em alimentos naturalmente contaminados, sendo que a $\mathrm{FB}_{1}$ é quase sempre a mais abundante, representando cerca de $70 \%$ da concentração total das fumonisinas detectadas (SYDENHAM $\boldsymbol{e} \boldsymbol{t} \boldsymbol{a l} .$, 1991; THIEL $\boldsymbol{e} \boldsymbol{t} \boldsymbol{a l}$., 1991).

A primeira descrição sobre a ocorrência natural de $\mathrm{FB}_{1}$ foi realizada por SYDENHAM et $\boldsymbol{a l}$. (1990) a partir de milho mofado colhido de uma área em Transkei, Sul da África, que apresentava alta incidência de câncer de esôfago em humanos. Os níveis detectados nas amostras variavam de 44 a $83 \mu \mathrm{g} / \mathrm{g}$. O milho proveniente de algumas regiões da África, com histórico da doença, apresentaram altos níveis de contaminação $(117 \mu \mathrm{g} / \mathrm{g})$, segundo análises realizadas por THIEL et al. (1992).

As fumonisinas causam leucoencefalomalácia em eqüinos (LEME) (MARASAS $\boldsymbol{e t} \boldsymbol{a l}$., 1988), edema pulmonar e hidrotórax em suínos (HARRINSON et al., 1990), hepatotoxicidade e hepatocarcinogenicidade em ratos (GELDERBLOM et al., 1991), provável câncer de esôfago em humanos (SYDENHAM et al., 1990) e leucoencefalomalácia em coelhos (BUCCI et al., 1996). Os níveis de $\mathrm{FB}_{1}$ detectados em alimentos associados à ocorrência de LEME na África do Sul e Estados Unidos chegaram a $8850 \eta \mathrm{g} / \mathrm{g}, 7700 \eta \mathrm{g} / \mathrm{g}$ e $7200 \eta \mathrm{g} / \mathrm{g}$, conforme relatos de SHEPHARD et al. (1990), WILSON et al. (1990), THIEL et al. (1991), respectivamente.

WILSON et al. (1990) correlacionaram a ocorrência de fumonisinas com o diagnóstico de LEME, na África do Sul, analisando amostras de 
milho mofado com concentrações de $\mathrm{FB}_{1}$ que variavam de 37 a $122 \mu \mathrm{g} / \mathrm{g}$. Neste mesmo ano, PLATTNER $\boldsymbol{e} \boldsymbol{t}$ al. (1990) e SHEPHARD $\boldsymbol{e} \boldsymbol{t} \boldsymbol{a}$. (1990) também detectaram $\mathrm{FB}_{1}$ e $\mathrm{FB}_{2}$ em amostras de milho destinado a eqüinos que apresentaram quadro típico de LEME nos Estados Unidos e África do Sul, respectivamente.

No Brasil, são freqüentes os relatos de leucoencefalomalácia eqüina (RIET-CORRÊA et al., 1982; HIROOKA et al., 1988; HIROOKA et al., 1991; HIROOKA \& YAMAGUCHI, 1994; MEIRELLES et al., 1992; MEIRELLES et al., 1994). As primeiras citações sobre a detecção $F_{B}$ e surtos de LEME ocorridos no país foram realizados por (MEIRELLES et al., 1991; HIROOKA et al., 1991; YAMAGUCHI et al., 1992; HIROOKA et al., 1996).

As fumonisinas foram detectadas naturalmente em vários tipos de alimentos, principalmente o milho e derivados, em vários países (Canadá, Egito, Peru, África do Sul, EUA), indicando a exposição do homem às micotoxinas em até $3 \mu \mathrm{g} / \mathrm{g}$ (SYDENHAM et al., 1991). Nos Estados Unidos, ROTTINGHAUS et al. (1992) encontraram concentrações de $\mathrm{FB}_{1}$ entre 0,1 a $5,0 \mu \mathrm{g} / \mathrm{g}$ em $15 \%$ das amostras de milho analisadas. Em outro trabalho, amostras de farelo de milho provenientes da mesma região produtora, os níveis detectados foram de até 2,8 $\mu \mathrm{g} / \mathrm{g}$ (HOLCOMB et al., 1993).

No Brasil, YAMAGUCHI et al. (1992) analisaram 39 lotes de milho colhidos na safra de 1990 e 1991, provenientes de quatro regiões produtoras no Estado do Paraná. A análise de fumonisinas por Cromatografia Líquida de Alta Eficiência (CLAE) foi positiva em 97,4\% das amostras para $\mathrm{FB}_{1}$ e $4,8 \%$ para $\mathrm{FB}_{2}$. As concentrações das micotoxinas detectadas no milho variaram, conforme a região, de 0,6 a $12,6 \mu \mathrm{g} / \mathrm{g}$ para $\mathrm{FB}_{1}$ e 0,0 a $10,4 \mu \mathrm{g} / \mathrm{g}$ para $\mathrm{FB}_{2}$

HIROOKA et al. (1996) analisaram 48 amostras de milho no Estado do Paraná e nove no Mato Grosso do Sul e Goiás, colhidas entre 1990 e 1991. Os pesquisadores detectaram fumonisinas em todas as amostras colhidas no Paraná, com níveis que variavam, de acordo com a região, de 3,25 a $4,79 \mu \mathrm{g} / \mathrm{g}$ de $\mathrm{FB}_{1}$ e 2,34 a $3,45 \mu \mathrm{g} / \mathrm{g}$ para $\mathrm{FB}_{2}$. Com exceção de uma amostra proveniente do Estado de Goiás, as outras provenientes da região Central do Brasil também estavam contaminadas com $\mathrm{FB}_{1}$ e $\mathrm{FB}_{2}(5,45$ e $5,0 \mu \mathrm{g} / \mathrm{g}$, respectivamente). Já no Rio Grande do Sul, MALLMANN et al. (1997) analisaram 169 amostras de alimentos entre os anos de 1996 e 1997 e verificaram a contaminação por fumonisinas em $47,1 \%$ das amostras de milho, com concentração média de $8,4 \mu \mathrm{g} / \mathrm{g}$.
Estudos realizados por ORSI et al. (2000) mostraram a ocorrência natural de fumonisinas em 195 amostras de híbridos de milho no Estado de São Paulo, sendo $90,2 \%$ delas positivas para $\mathrm{FB}_{1}$ e $97,4 \%$ para $\mathrm{FB}_{2}$. Os índices médios de contaminação foram de 9,72 $\mu \mathrm{g} / \mathrm{g}$ de $\mathrm{FB}_{1}$ e $7,67 \mu \mathrm{g} / \mathrm{g}$ de $\mathrm{FB}_{2}$. Diferenças regionais na concentração de fumonisina foram encontradas quando o mesmo milho híbrido foi analisado no Estado do Paraná, indicando interferências climáticas na predominância de linhagens toxigênicas de Fusarium ONO et al. (2001)

\section{EFEITOS BIOLÓGICOS DAS FUMONISINAS}

A Leucoencefalomalácia eqüina (LEME) é uma doença não infecciosa esporádica e altamente fatal que afeta o SNC de cavalos e outros eqüídeos. A LEME foi descrita no início do século por BUCLEY \& McCALLUM (1901) e caracterizada por encefalite hemorrágica aguda acometendo os eqüinos de Maryland (EUA). BUTLER (1902) foi quem confirmou experimentalmente a doença, após a administração oral de milho mofado a eqüinos. Em 1971, WILSON \& MARANPOT (1971) conseguiram reproduzir experimentalmente a doença através da administração oral a eqüinos de milho contaminado artificialmente com Fusarium Verticillioides (Sacc.) Nirenb., isolados de cereal envolvido com surto da doença no Egito. No Brasil a doença foi descrita pela primeira vez em São Paulo por REGO (1950), entretanto a relação entre LEME e Fusarium verticillioides (Sacc.) Nirenb. foi estabelecida por RIET-CORREA et al. (1982) quando da descrição de três surtos da toxicose no Rio Grande do Sul.

Em 1988, após a descoberta das fumonisinas, realizada por GELDERBLOM et al. (1988), MARASAS et al. (1988) conseguiram reproduzir experimentalmente a LEME em eqüino que recebeu $0,125 \mathrm{mg} / \mathrm{kg}$ PV de FB1 purificada por via endovenosa durante nove dias (dose total: 276mg). Os sinais clínicos da micotoxicose tornaram-se aparentes no $8^{\underline{0}}$ dia após a inoculação e consistiam de apatia, tremores musculares, paresia do lábio inferior e língua, convulsões tetânicas e morte. À necropsia, foram observados edema cerebral acentuado e necrose focal bilateral da medula oblonga. As alterações bioquímicas observadas no soro sangüíneo foram pequena elevação das enzimas aspartato transferase (AST) e gama glutamil transferase (GGT).

A confirmação do papel da FB1 pura na apresentação dos sintomas foi realizada por KELLERMAN et al. (1990). Neste trabalho, os 
autores induziram LEME através da administração oral de FB1 a dois eqüinos, sendo que um deles recebeu FB1 (50\% de pureza) em 21 doses de $1,25 \mathrm{mg}$ a $4 \mathrm{mg} / \mathrm{kg} / \mathrm{PV}$ durante período de 33 dias (dose total de $8,925 \mathrm{~g}$ ) e o outro animal recebeu $1 \mathrm{a}$ $4 \mathrm{mg} / \mathrm{kg} / \mathrm{PV}$ de FB1 pura durante 24 dias (dose total de $8,417 \mathrm{~g})$. Entre o $24^{\circ}$ e o $30^{\circ}$ dias após a dosificação oral, os animais desenvolveram sinais neurotóxicos que incluíam alterações no comportamento, incoordenação, paralisia dos lábios e língua e tremores musculares. Após a eutanásia, realizada no início do aparecimento dos sinais, os autores observaram lesões típicas da doença com a presença de necrose cavitária ou amolecimento da substância branca de ambos hemi sférios cerebrais.

Em suínos, o consumo de milho contaminado por $F$. verticillioides (Sacc.) Nirenb. na safra de 1989, nos EUA, levou ao aparecimento de uma doença caracterizada por severo edema pulmonar e hidrotórax. HARRISON et al. (1990) colheram milho de duas fazendas, onde 34 suínos adultos morreram de edema pulmonar, cinco dias após o consumo de alimento contaminado. Um fungo morfologicamente idêntico ao $\boldsymbol{F}$. verticillioides (Sacc.) Nirenb. foi isolado e teores de 105 a $155 \mathrm{mg} / \mathrm{kg}$ de $\mathrm{FB}_{1}$ foram detectados no alimento. As alterações patológicas observadas nos animais mortos indicavam edema pulmonar $\mathrm{e}$ hidrotórax. No mesmo estudo, os autores injetaram, por via endovenosa, $\mathrm{FB}_{1}$ purificada em dois animais nas doses de 0,4 a $0,174 \mathrm{mg} / \mathrm{kg}$ pc. $\mathrm{O}$ animal que recebeu a maior dose morreu no $5^{\underline{0}}$ dia após a inoculação, com lesões típicas de edema pulmonar.

O aparecimento de lesões e sinais clínicos nos suínos está relacionado à quantidade de toxina ingerida e dias de consumo (HASCHEK $\boldsymbol{e t} \boldsymbol{a l}$., 1992). Os sinais clínicos que incluem inapetência, depressão, icterícia e síndrome hepática ocorrem cinco dias após a ingestão de ração contaminada com $20 \mu \mathrm{g} / \mathrm{g}$ de $\mathrm{FB}_{1}$. A taxa de mortalidade pode estar em torno de 50\% (OSWEILER et al., 1992). Observa-se aumento dos níveis sanguiíneos de colesterol, bilirrubina e das atividades das enzimas aspartato aminotransferase e gama glutamiltranspeptidase (OSWEILER et al., 1992; MOTELIN et al., 1994). A morte de suíno intoxicado ocorre normalmente poucas horas após o início da apresentação dos sintomas. Na necropsia, pode ser observado edema pulmonar, hidrotórax, icterícia e fígado escurecido e firme. Doses baixas induzem degeneração hepática progressiva, enquanto doses mais altas causam edema pulmonar (OSWEILER $\boldsymbol{e t} \boldsymbol{a l} .$, 1992). Como em outras espécies animais, as fumonisinas alteram a biossíntese dos esfingolipídeos, com as maiores alterações nas concentrações das bases esfingoides no rim, fígado, pulmão e coração. O sistema imune específico não é afetado, entretanto $\mathrm{FB}_{1}$ inibiu a fagocitose e a biossíntese de esfingolipídeos nos macrófagos pulmonares, induzindo um acúmulo de material membranoso nas células endoteliais dos capilares pulmonares. Essa alteração parece ser específica a esse tipo de célula e a espécie suína (HASCHEK $\boldsymbol{e t}$ al., 2001).

Estudos sobre os efeitos tóxicos das fumonisinas em aves foram conduzidos utilizando material de cultura de $\boldsymbol{F}$. verticillioides (Sacc.) Nirenb. como fonte de contaminação. LEDOUX $\boldsymbol{e t}$ al. (1992) alimentaram pintinhos de um dia com dietas contendo níveis de 0, 100, 200, 300 ou 400ppm de $\mathrm{FB}_{1}$, durante 21 dias. $\mathrm{O}$ ganho diário de peso diminuiu com o aumento do nível de $\mathrm{FB}_{1}$ na dieta. Lesões histopatológicas indicaram atrofia do timo, hiperplasia biliar e necrose hepática

HENRY \& WYATT (1994) estudaram a toxicidade da fumonisina $\mathrm{B}_{1}$ purificada em frangos em crescimento. A toxina foi incorporada na dieta de pintinhos de 1 dia nas concentrações de 0, 20, 40 e $80 \mathrm{ppm}$. Níveis de até $80 \mathrm{ppm}$ não alteraram o ganho de peso, conversão alimentar e consumo de água. Nenhuma diferença quanto ao peso dos órgãos (fígado, baço e rins) foi observada. Os autores concluíram que dietas com níveis de até 80ppm não alteram o desempenho de frangos. Entretanto, os autores salientam a necessidade de pesquisas sobre a interação das fumonisinas com outras micotoxinas .

Os bovinos parecem ser menos susceptíveis aos efeitos adversos de $\mathrm{FB}_{1}$. OSWEILER et al. (1993) avaliaram os efeitos da administração de material de cultura de $\boldsymbol{F}$. verticillioides (Sacc.) Nirenb. incorporado à dieta de 18 bezerros. Os alimentos continham 15, 31, 148mg de $\mathrm{FB}_{1} / \mathrm{kg}$ e foram administrados durante o período de 30 dias. Os autores não observaram alterações no desempenho dos animais e houve apenas alterações das enzimas hepáticas (AST, GGT e lactato desidrogenase). O fornecimento aos bezerros, de dietas com níveis de $\mathrm{FB}_{1}$ considerados tóxicos a equiinos e suínos, não causaram alterações significativas nestes animais.

Estudos sobre a presença de $\mathrm{FB}_{1}$ no leite sugerem que a contaminação por esta micotoxina pode ser teóricamente possível. Entretanto, alguns autores já observaram, após a administração de $\mathrm{FB}_{1}$ por via oral e intravenosa a vacas leiteiras, a não detecção de resíduos da toxina no leite (SCOTT $\boldsymbol{e t}$ al., 1994; RICHARD et al., 1996). 


\section{MECANISMO DE AÇÃO DAS FUMONISINAS}

O mecanismo de ação das fumonisinas ainda não é perfeitamente conhecido, mas WANG $\boldsymbol{e t}$ al. (1991), propuseram que a FB1 poderia intervir na biossíntese de esfingolipídios ou "turnover" de esfingosina, porque existe uma similaridade da molécula de FB1 com o complexo amino álcool esfingosina, que é um dos trinta ou mais aminoalcoois da cadeia longa encontrados nos esfingolipídeos de várias espécies. Os esfingolipídeos mais abundantes dos tecidos animais são a esfingosina e os glicoesfingolipídeos. A esfingomielina é um fosfolipideo que contém ácido graxo, fosfato, colina e o amino álcool complexo esfingosina.

A inibição de biossíntese dos esfingolipídeos pode ter um profundo efeito sobre a célula, uma vez que esses componentes têm papel importante na estrutura da membrana, comunicação celular, interação intracelular e matrix celular, regulação de fatores de crescimento, como mensageiro de vários fatores, incluindo fator de necrose de tumor, interleucina 1 e fator de crescimento de nervos (MERRILL et al., 1993).

A esfingosina é sintetizada no retículo endoplasmático através da combinação de palmitoylCoA e aminoácido serina, formando o 3-ceto esfingonina que é reduzido à esfinganina $\mathrm{e}$ esfingosina. Ceramida é formada por combinação ou de um ácido graxo livre ou acetil CoA e esfingosina. Esfingomielina é formada quando ceramida reage com CDP - colina ou fosfatidil colina. Para testar a hipótese de que fumonisina age alterando a biossíntese de esfingolípideos, WANG et al. (1991) examinaram os efeitos da $\mathrm{FB}_{1}$ sobre a habilidade de hepatócitos de ratos converter serina [C] aos esfingolipídeos. Mostrou-se que $\mathrm{FB}_{1}$ inibiu a biossíntese "de novo" de esfingolípideos com uma $\mathrm{K}_{50}$ de 0,1um. Assim, as fumonisinas parecem ter uma ação contra a biossíntese de esfingolipeos em concentrações que poderiam ser atingidas pelo consumo de milho naturalmente contaminado.

$\mathrm{O}$ sitio específico de ação de $\mathrm{FB}_{1}$ parece ser as enzimas esfinganina e esfingosina $\mathrm{N}$ acetiltransferase. A inibição desta via metabólica resulta na depleção do complexo esfingolipídeo, aumento intracelular de concentração de esfinganina livre (ou em menor grau de esfingosina livre) e um aumento dos produtos de clavagem (ABBAS et al., 1993). O acúmulo das bases esfingoides é a causa primária da toxicidade das fumonisinas. Apesar disso, os plenos efeitos das fumonisinas provavelmente envolvem muitos eventos bioquímicos. A elevação nas bases esfingóides também afetam a quantidade de outros lipídeos, incluindo os derivativos 1-fosfatos e Nacetil de esfinganina. Além disso, o aminopentol de $\mathrm{FB}_{1}(\mathrm{AP}(1))$ é um inibidor e substrato para a enzima ceramida sintase e a molécula resultante, $\mathrm{N}$ palmitoil-AP(1)(PAP(1)) é um potente inibidor da enzima e dez vezes mais tóxica do que a $\mathrm{FB}_{1}$ ou AP(1) para as células HT-29 em cultura (MERRILL et al., 2001). A alteração no metabolismo dos esfingolípideos pode ser monitorada, já que alguma esfinganina que acumula na célula pode aparecer no sangue periférico. WANG et al. (1992) alimentaram pôneis com dietas contendo $44 \mu \mathrm{g} / \mathrm{g}$ de FB1 durante 10 dias e observaram, no vigésimo dia do experimento, elevação no nível de esfinganina $(2,7$ vezes). As elevações das enzimas séricas ocorreram somente após 10 dias, indicando que os níveis de esfinganina e esfingosina podem ser utilizados como marcadores de exposição animal as fumonis inas.

\section{CONCLUSÃO}

As fumonisinas são micotoxinas produzidas por fungos do gênero Fusarium e detectadas em grande frequiência em grãos de milho no Brasil e no mundo. Considerando que esse cereal é matéria-prima básica na formulação de rações destinadas a animais de produção e que estudos de biodisponibilidade realizados em suínos detectaram resíduos do metabolismo da $\mathrm{FB}_{1} \mathrm{em}$ tecidos desses animais, estudos são necessários para melhorar os métodos de detecção das bases enfingóides esfinganina e esfingosina, para monitoramento da exposição dos animais e do homem a essas micotoxinas.

\section{REFERÊNCIAS BIBLIOGRÁFICAS}

ABBAS, H.K., DUKE, S.O., TANAKA, T. Phitotoxicity of fumonisin and related compounds. J Toxicol - Toxin Reviews, v.12, p. 225-251, 1993.

ABBAS, H.T., OCAMB, C.M. First report of fumonisin $\mathrm{B}_{1}$ Fusarium polyphialidicum collected from seeds of Pinus strobes. Plant Dis, v.79, p.642-645, 1995.

AH SEO, J., WON LEE, Y. Natural occurrence of the C series of fumonisins in moldy corn. Appl Environm Microbiol, v.65, p.1331-1334, 1999

ALLCROFT, R., CARNAGHAN, R.B.A., SARGENT, K., et al A toxic factor in Brazilian groundnut meal. Vet Rec, v.73, p.128-129, 1961.

ALLCROFT, P., CARNAGHAN, R.B.A. Groundnut toxicity Aspergillus flavus toxin (aflatoxin) in animal products. Preliminary Comunication. Vet Rec, v.74,p.863-864, 1962. 
ASEVEDO, I.G., GAMBALE, W., CORRÊA, B., et al. Mycoflora and aflatoxicogenic species of Aspergillus spp isolated from stored maize. Rev Microbiol, v.25, p.46-50, 1994.

BEZUIDENHOUT, S.C., GELDERBLOM, W.C.A., GORST ALLMAN, C.P., et al. Structure elucidation of the fumonisins, mycotoxins from $\mathbf{F u s a r i u m ~ m o n i l i f o r m e . ~} \mathbf{J}$ Chem Soc Chem Commun, p.743-745, 1988.

BRANHAM, B.E., PLATTNER, R.D. Isolation and characterization of a new fumonisin from liquid cultures of Fusarium moniliforme. J Nat Prod, v.56, p.1630-1633, 1993

BUCCI, T., HANSEN, D.K., LaBORDE, J.B Leukoencefalo malacia and hemorrhage in the brain of rabbits gavaged with mycotoxin fumonisin $\mathrm{B}_{1}$. Nat Toxins, v. 4 p.51-52, 1996 .

BUCKLEY, S.S., MAcCALLUM, W.G. Acute hemorrhagii encephalitis prevalent among horses in Maryland. Am Vet Rev, v.25, p.99-101, 1901

BUTLER, T. Notes on a feeding experiment to produce leucoencephalitis in a horses with positive results. Am Vet Rev, v.26, p.748-751, 1902.

CAWOOD, M.E., GELDERBLOM, W.C.A., VLEGGAAR, R., $\boldsymbol{e}$ al. Isolation of the fumonisin mycotoxins: a quantitative approach. J Agric Food Chem, v.39, p.1958-1962, 1991

GELDERBLOM, W.C.A., JASKIEWICZ, K., MARASAS, W.F.O. et al. Fumonisins: novel mycocotoxin with cancerpromoting activity produced by Fusarium moniliforme. Appl Environm Microbiol, v.54, p.1806-1811, 1988.

GELDERBLOM, W.C.A., JASKIEWICZ, K., MARASAS, W.F.O. et al. Toxicity and carcinogenicity of the Fusarium moniliforme metabolite, fumonisin B1, in rats. Carcinogenesis, v.12, p.1247-1251, 1991

HARRISON, L.R., COLVIN, B.M., GREENE, J.T., et al. Pulmonary edema and hidrothorax in swine produced by fumonisin B1 a toxic metabolite of Fusarium moniliforme. J Vet Diagn Invest, v.2, p.217-221, 1990

HASCHEK, W.M., MOTELIN, G., NESS, D.K., et al. Characterization of fumonisin toxicity in orally and intravenously dosed swine. Mycopathol, v.117, p. 83-96, 1992.

HASCHEK, W.M., GUMPRECHT, L.A., SMITH, G., et al Fumonisin toxicosis in swine: An overview of porcine pulmonary edema and current perspectives. Environm Health Perspect, v.109, p. 251-257, 2001.

HENRY, M.H. WYATT, R.D. A review of fumonisin production by Fusarium moniliforme and fumonisin toxicoses in animals. Appl Poult Sci, v.2,p. 188-192, 1994.

HIROOKA, E.Y., VIOTTI, N.M.A., SOARES, L.M.V., et al. Intoxicação em eqüinos por micotoxinas produzidas por Fusarium moniliforme no Norte do Paraná. Semina Ci Agri, v.9, p.128-135, 1988

HIROOKA, E.Y., SHIBATA, M.M., VIOTTI, N.M.A., $\boldsymbol{e}$ t al Fumonisina: importância da nova micotoxina de Fusarium moniliforme em intoxicações animais no Norte do Paraná. Rev de Microbiol, v.22, p.312, 1991
HIROOKA, E.Y., YAMAGUCHI, M.M. Micotoxinas e metabólitos bioativos de Fusarium: perspectiva de sua importância para o Brasil. Semina Ci Agr, v.15, p.74-79, 1994.

HIROOKA, E.Y., YAMAGUCHI, M.M., AOYAMA, S., et al. The natural occurrence of fumonisin in Brazilian corn kernels. Food Addit Contam, v.13, p.173-183, 1996.

HOLCOMB, M., SUTHERLAND, J.B., CHIARELLI, M.D. HPLC and FAB mass spectrometry analysis of fumonisin B1 and B2 produced by Fusarium moniliforme on food substrates. J Agric Food Chem, v.41, p.357-360, 1993.

KELLERMAN, T.S., MARASAS, W.F.O., THIEL, P.G., et al. Leukoencephalomalacia in two horses induced by oral dosing of fumonisin B1. Onderstepoort J Vet Res, p. 269-275, 1990.

LEDOUX, D.R., BROWN, T.P., WEIBKING, T.S., $\boldsymbol{e}$ t al. Fumonisin toxicity in broiler chicks. J Vet Diagn Invest, v.4, p.330-333, 1992 .

MALLMANN, C., SANTURIO, J.M., DILKIN, P., et al. Incidência de fumonisina $B_{1}$ em milho e rações no Brasil. In: CONGRESSO LATINO AMERICANO DE MICOTOXICOLOGIA, 7, 1997, Maracay, Venezuela. Anais... Maracay : Sociedad LatinoAmericana de Micotoxicologia, 1997. p.73

MARASAS, W.F.O., KELLERMAN, T.S., GELDERBLOM, W.C.A., et al. Leukoencephalomalacia in horse induced by fumonisin B1 isolated from Fusarium moniliforme. Onderste poort J Vet Res, v. 55, p.197-203, 1988.

MEIRELLES, M.C.A., CORRÊA, B., FISCHMAN, O., et al. Leucoencefalomalacia (LEME) no Brasil I- Aspectos epizotiológicos e micotoxicológicos dos surtos ocorridos nos anos de 1988 a 1990. Rev Microbiol, v.22, p. 316, 1991.

MEIRELLES, M.C.A., CORRÊA, B., FISCHMAN, O., et al. Leucoencefalomalácia equina (LEME) no Brasil. II-Aspectos microbiológicos e micotoxicológicos dos surtos ocorridos nos anos de 1988 a 1990. In: ENCONTRO NACIONAL DE MICOTOXINAS, 7, São Paulo, 1992. Anais... São Paulo, Instituto Adolfo Lutz, 1992. p.18

MEIRELLES, M.C.A., CORRÊA, B., FISCHMAN, O., et al. Mycoflora of the toxic feeds associated with equine leucoencephalomalacia (ELEM) outbreaks in Brazil. Mycopathol, v.117, p. 183-188, 1994.

MERRILL, A.H., VAN ECHTEN, G., WANG, E., $\boldsymbol{e}$ t al Fumonisin $B_{1}$ inhibits sphingosine (sphinganine) N acyltransferase and de novo sphingolipid biosynthesis in cultured neuron in situ. J Biol Chem, v.268, p.2299-2306, 1993.

MERRILL, A.H., SULLARDS, M.C., WANG, E., et al. Sphingolipid metabolism: roles in signal transduction and disruption by fumonisins. Environm Health Perspect, v.109, p. 283-289, 2001.

MILLS, J.T. Ecology of mycotoxigenic Fusarium species on cereal seeds. J Food Prot, v.52, p.737-742, 1989.

MOSS, M.O. Economic importance of mycotoxins-recent incidence in the United States. Anim Sci, v.27, p. 39413949, 1991. 
MOTELIN, G.K., HASCHEK, W.M., NESS, D.K., et al. Temporal and dose response features in swine fed corn screenings contamined with fumonisin mycotoxins Mycopathol, v. 126, p. 27-40, 1994.

MUSSER, S.M., PLATTNER, R.D. Fumonisin composition in culture of Fusarium moniliforme, Fusarium proliferatum and Fusarium nygamae J Agri Food Chem, v.45, p. 1169$1173,1997$.

NELSON, P.E. Taxonomy and biology of Fusarium moniliforme. Mycopathol, v.117, p.29-36, 1992.

ONO, E.Y.S., ONO, M.A., FUNO, F.Y., et al. Evaluation of fumonisin-aflatoxin-co-occurrence in Brazilian corn hybrids by ELISA. Food Addit Contam, v.18, n.8., p. 719-729, 2001.

ORSI, R.B., CORRÊA, B., POZZI, C.R., et al. Mycoflora and occurrence of fumonisins in freshly harvested and stored hybrid maize. J Stored Prod Res, v.36, p. 75-87, 2000

OSWEILER, G.D., ROSS, P.F., WILSON, T.M., et al. Characterization of an epizootic of pulmonary edema in swine in corn screening. J Vet Diagn Invest, v.4, p.53-59, 1992

OSWEILER, G.D., KEHRLI, M.E., STABEL, J.R, et al. Effects of fumonisin-contaminated corn screenings on growth and health of feeder calves. J Anim Sci, v.71, p. 459-466, 1993.

PLATTNER, R.D., NORRED, W.P., BACON, C.W., et al. A method of detection of fumonisins in corn samples associated with field cases of equine leukoencephalomalacea. Mycologia, v. 82, p. 692-702, 1990.

POZZI, C.R., CORRÊA, B., GAMBALE, W., et al. Postharvest and stored corn in Brazil: mycoflora interation, abiotic factors and mycotoxins occurence. Food Addit Contam, v.12, n.3 p. 313-319, 1995

REGO, C.L. Doença de sintomatologia nervosa causada por intoxicação pelo milho. Rev Mil Remonta Vet, v.10, p.199$215,1950$.

RICHARD, J.L., MEERDINK, G., MARAGOS, C.M., et al. Absence of detectable fumonisin in the milk cows fed Fusarium proliferatum (Nirenberg) culture material. Mycophatol, v.133, p.123-126, 1996

RIET-CORREA, F., MEIRELES, M.C.A., SOARES, J.M., et $a l$. Leucoencefalomalácia em eqüinos associada à ingestão de milho mofado. Pesq Vet Bras, v.2, p.27-30, 1982.

ROSS, P.F., NELSON, P.E., RICHARD, I.D., et al. Production of fumonisins by Fusarium moniliforme and Fusarium proliferatum isolate associated wirh equine leukoencephalomalacia and pulmonary edema syndrome in swine. Appl Environm Microbiol., v.56, p. 3225-3226, 1990.

ROTTINGHAUS, G.E., COATNEY, C.G., MINOR, H.C. A rapid, sensitive thin layer chromatography procedure for the detection of fumonisin B1 and B2. J Vet Diagn Invest v.4, p.326-330, 1992.
SCOTT, P.M. Fumonisins. Inst. J Microbiol, v.18, p.257-270, 1993.

SCOTT, P.M., DELGADO, T., PRELUSKY, D.B., et al. Determination of fumonisins in milk. J Environm Sci Health, v.29, p. 989-998, 1994

SHEPHARD, G.S., SYDENHAM, E.W., THIEL, P.G., et al. Quantitative determination of fumonisins B1 and B2 by highperformance liquid chromatography with fluorescence detection. J Liq Chromatogr, v.13, p.2077-2087, 1990.

SILVA, J.B., POZZI, C.R., MALLOZZI, M.A.B., et al. Mycoflora and occurrence of aflatoxin $\mathrm{B}_{1}$ and fumonisin $\mathrm{B}_{1}$ during storage of brazilian sorghum. J Agri Food Chem, v.48, p. 4352-4356, 2001.

SYDENHAM, E.W., GELDERBLOM, W.C.A., THIEL, P.G., $\boldsymbol{e}$ al. Evidence for the natural occurrence of Fumonisin B1, a mycotoxin produced by Fusarium moniliforme in corn. J Agric Food Chem, v.38, p.285-290, 1990.

SYDENHAM, E.W., GELDERBLOM, W.C.A., THIEL, P.G., $\boldsymbol{e}$ al. Evidence for the natural occurrence of fumonisin $\mathrm{B}_{1}$ a mycotoxin produced by $\mathbf{F u s a r i u m ~ m o n i l i f o r m e ~ i n ~ c o r n . ~} \mathbf{J}$ Agric Food Chem, v.39, p.2014-2018, 1991

THIEL, P.G., SHEPHARD, G.S., SYDENHAM, E.W., et al. Levels of fumonisins B1 and B2 in feeds associated with confirmed cases of equine leukoencephalomalacia. J Agric Food Chem, v.39, p.109-111, 1991.

THIEL, P.G., MARASAS, W.F.O., SYDENHAM, E.W., $\boldsymbol{e}$ t al. The implications of naturally occurring levels of fumonisins in corn human and animal health. Mycopathol, v.117, p.3-9, 1992.

WANG, E., NORRED, W.P., BACON, C.W., et al. Inhibition of sphingolipid biosynthesis by fumonisins implications for diseases associated with Fusarium moniliforme. J Biol Chem, v.266, p.1486-1490, 1991.

WANG, E., ROSS, F.P., WILSON, T.M., et al. Increases in serum sphingosine and sphinganine and decreases in complex sphingolipids in ponies, given, feed containing fumonisins, mycotoxins produced by Fusarium moniliforme. Nutrit Pharmacol Toxicol, v.122, p.1706-1716, 1992.

WILSON, B.J., MARONPOT, R.R. Causative fungus agent of leukoencephalomalacia in equine animals. Vet Rec, v.88, p.484-486, 1971.

WILSON, T.M., ROSS, P.F., RICE, L.G., et al. Fumonisin B levels associated with and epizootic of equine leukoencephalomalacia. J Vet Diagn Invest, v.2, p. 213-216, 1990.

YAMAGUCHI, M.M., HIROOKA, E.Y., SHIBATA, T.M.M., $\boldsymbol{e}$ al. Fumonisinas em milho no Estado do Paraná. In: ENCONTRO NACIONAL DE MICOTOXINAS 7, São Paulo, 1992. Anais... São Paulo : Instituto Adolfo Lutz, 1992. p. 27 